Check for updates

Cite this: RSC Adv., 2017, 7, 22954

\title{
Surface spermidine functionalized PEGylated poly(lactide-co-glycolide) nanoparticles for tumor-targeted drug delivery $\dagger$
}

\author{
Jianming Li, (iD a Juan Mao, ${ }^{a}$ Jing Tang, ${ }^{\mathrm{b}}$ Guo Li, ${ }^{a}$ Fengling Fang, ${ }^{\mathrm{a}}$ Yana Tang ${ }^{\mathrm{a}}$ \\ and Jinsong Ding*a
}

Development of targeted drug delivery systems for tumor therapy provides an effective approach to minimize the side effects originating from arbitrary in vivo drug distribution after systemic administration. In this study, we reported DOX-loaded PEG-PLGA nanoparticles with the surface modified with spermidine (SPD) (SPD-DOX-NPS) for targeted delivery of DOX into tumor cells, which benefits from the polyamine transport system (PTS) on the tumor cell surface. The as-prepared NPs showed a typical spherical morphology with about $150 \mathrm{~nm}$ diameter and near electric neutrality of surface charge. The in vitro drug release displayed a sustained release profile. Characterization by fluorescence microscopy and flow cytometry revealed that the cellular uptake of SPD-DOX-NPs in A549 cells depends on SPD content on the surface of the particles. The uptake efficiency can be further increased by adding eflornithine (DMFO) and competitively inhibited by free SPD pre-treatment. These results collectively suggest that PTS is essential for particle internalization. Furthermore, the SPD-DOX-NPs exhibited significantly enhanced cytotoxicity towards A549 cells than the free DOX solution and SPD-free DOXNPs. Taken together, our results demonstrate that SPD-functionalized nanoparticles are a potential

Received 27th February 2017 Accepted 20th April 2017

DOI: $10.1039 / c 7 r a 02447 a$

rsc.li/rsc-advances candidate for targeted delivery of drugs into tumor cells.

\section{Introduction}

Cancer is a major public health problem with increasing incidence and mortality. ${ }^{1,2}$ While significant efforts have been made globally for the treatment of cancer over the past 50 years, it still remains the second-leading cause of death worldwide. ${ }^{3}$ Although chemotherapeutic agents are commonly used in the clinic to treat cancer, the inevitable side effects have required the improvement of antineoplastic therapies to prolong the survival time of patients in medical intervention. ${ }^{4}$

With the development of nanomedicine, the past two decades have witnessed a rapid development of nanotechnology-based drug delivery systems as they provide an alternative therapy for progressive and long-term delivery of therapeutic agents, such as liposomes, micelles, solid lipid nanoparticles (SLNs) or polymeric nanoparticles. ${ }^{5,6}$ The design methodology of novel drug delivery systems is considered to be the key part of targeting and localized delivery of chemotherapy

${ }^{a}$ Xiangya School of Pharmaceutical Sciences, Central South University, Changsha
410013, Hunan, China. E-mail: dingjs0221@163.com; Fax: +86-731-82650442; Tel:
$+86-731-82650250$
${ }^{b}$ Department of Pharmaceutics, Changsha Medical University, Changsha 410219,
Hunan, China $\dagger$ Electronic supplementary information (ESI) available. See DOI: $10.1039 / \mathrm{c} 7 \mathrm{ra} 02447 \mathrm{a}$ drugs to the cancer tissues. Compared with conventional formulations, the therapeutic agents encapsulated in nanoparticles possess characteristics of desired solubility, enhanced bioavailability and selective biodistribution endowed by their carriers. Among the carriers, the nanoparticles (NPs) prepared from poly (lactide-co-glycolide) (PLGA) have been extensively used due to its biocompatible, biodegradable and nontoxicity, which have also been demonstrated to be advantageous for sustained and controlled drug release with clinical safety. ${ }^{7,8}$ Further modification of such particles with poly (ethylene glycol) (PEG) could enhance the systemic retention, since it can reduce nonspecific protein adsorption, opsonization and subsequent clearance by the reticuloendothelial system (RES). As a result, the improved drug accumulation within tumor tissues can be expected via the enhanced permeability and retention (EPR) effects. ${ }^{9,10}$

However, one major limitation of PLGA-NPs is that it cannot differentiate the cancerous cells from normal cells. Recent advances have indicated that the particles with surface modified by targeting ligands, such as aptamers, antibodies, peptides and integrin, could actively internalize into cancerous via ligands/ acceptors (overexpressed on tumor cells) interactions. ${ }^{11-13}$ Herein, we focused on a specific type of ligand, namely polyamines (PAs), such as putrescine (PUT), spermine (SPM) and spermidine (SPD), which are small molecular aliphatic amines involved in cell cycle, apoptosis and autophagy. ${ }^{14}$ 
It is known that various tumor cells, such as neuroblastoma, leukemia, breast cancer, lung cancer and melanomas, overexpress the polyamine transport system (PTS, a specific energydependent transporter or ion channel) on their surface to transport exogenous PAs into cells. ${ }^{15,16}$ It shows that PTS may offer an alternative targeting delivery strategy as PAs (or PAs analogue)-drug conjugates can selectively penetrate into tumor cells via the molecular recognition events involved in polyamine skeleton. An additional benefit is that chemical conjugation of PAs could effectively improve the solubility of chemotherapeutics. ${ }^{17-19}$ However, the PTS mediated antineoplastic drug loaded targeting delivery systems have yet been explored. As PAs possess extraordinary binding affinity and specificity to PTS, ${ }^{20}$ we reason that PAs-modified NPs holds great potential as a promising vector for anti-tumor therapy.

Doxorubicin (DOX), an anthracycline derivative, is one of the most successful first-line chemotherapeutic anticancer drugs with broad spectrum effectiveness in clinical application against multiple cancers. ${ }^{21}$ However, the significant curative effect of DOX is often associated with unrestricted biodistribution and undesirable systemic toxicity to healthy tissues. $^{22,23}$ Therefore, the design of targeted DOX delivery systems to inhibit the low bioavailability, rapid drug clearance and serious side effects (such as cardiotoxicity) is critically desired. ${ }^{24}$

In this study, we utilized SPD, a natural polyamine with moderate chain length and positive charge, as targeting ligand to prepare PAs conjugated, DOX-loaded PEG-PLGA (SPD-DOXNPs) nanoparticles, aiming to actively deliver DOX to cancer cells via the specific binding between SPD and PTS. The constructed SPD-modified nanoparticles were characterized with average particle size, zeta potential, surface morphology, drug encapsulation efficiency (EE), drug loading capacity (LC) and in vitro drug release, respectively. Cellular uptake and in vitro antitumor efficacy of SPD-DOX-NPs for A549 cancer cells were then investigated.

\section{Materials and methods}

\subsection{Materials}

Carboxyl-terminated poly(D,L-lactic-co-glycolic acid) (PLGA$\mathrm{COOH}$ ) (average $M_{\mathrm{w}}: 20000 \mathrm{Da}$; lactic acid: glycolic acid = 50 : 50) was purchased from Daigang Biomaterial Co., Ltd. (Jinan, China). $\mathrm{NH}_{2}-\mathrm{PEG}-\mathrm{COOH}\left(M_{\mathrm{w}}\right.$ : 3400) was obtained from Seebio Biotech, Co., Ltd. (Shanghai, China). $N$-(3-Dimethylaminopropyl)- $N^{\prime}$-ethylcarbodiimide hydrochloride $(\mathrm{EDC} \cdot \mathrm{HCl})$ and $N$-hydroxysuccinimide (NHS) were obtained from Qiyun Biotech, Co., Ltd. (Guangzhou, China). Spermidine (SPD), poly(vinyl alcohol) (PVA, 87-90\% hydrolyzed, $M_{\mathrm{w}}$ : $30000-$ $70000), \quad N, N$-diisopropylethylamine (DIEA) and 3-[4,5dimethylthiazol-2-yl]-2,5-diphenyl tetrazolium bromide (MTT) were obtained from Sigma-Aldrich (MO, USA). 1,5-Bis-Boc1,5,10-spermidine (Boc-SPD) was purchased from Chem-Impex Int'l. Inc., (IN, USA). Doxorubicin (DOX) was obtained from Macklin Biotech, Co., Ltd. (Shanghai, China). All the other solvents were analytical or chromatographic grade.
Cell lines: A549 cells were obtained from XiangYa Central Experiment Laboratory (Hunan, China). Dubelcco's Modified Eagle's Medium (DMEM), fetal bovine serum (FBS) were purchased from GIBCO (Grand Island, NY, USA). TrypsineEDTA, penicillin-streptomycin solution and 4',6-diamidino-2phenylindole (DAPI) were provided by Solarbio Biotech, Co., Ltd. (Beijing, China).

\subsection{Synthesis of PLGA-PEG-SPD block copolymer}

PLGA-COOH (4 g, $0.2 \mathrm{mmoL}$ ) dissolved in dichloromethane (DCM, $10 \mathrm{~mL}$ ) was activated by EDC (307 mg, $1.6 \mathrm{mmoL})$ and (NHS, $186 \mathrm{mg}, 1.6 \mathrm{mmoL}$ ) at RT under nitrogen atmosphere for $12 \mathrm{~h}$. The resultant solution was precipitated and repeatedly washed in ice-cold diethyl ether and diethyl ether-methanol (7/ $3, \mathrm{v} / \mathrm{v}$ ), respectively. After dried under vacuum, the activated PLGA-NHS ( $1 \mathrm{~g}, 0.05 \mathrm{mmoL})$ dissolved in DCM ( $4 \mathrm{~mL})$ was followed by the addition of DIEA (26 mg, $0.2 \mathrm{mmoL}$ ) and $\mathrm{NH}_{2}-$ PEG-COOH (210 mg, $0.06 \mathrm{mmoL}$ ) dissolved in DCM (2 mL) in a drop-wise manner with gentle stirring. After reacted at RT under nitrogen atmosphere for $24 \mathrm{~h}$, the reaction was precipitated and repeatedly washed in ice-cold diethyl ether and diethyl ether-methanol (7/3, v/v), respectively. And the di-block copolymer PLGA-PEG-COOH was collected and dried.

SPD-functionalized copolymer SPD-PEG-PLGA was synthesized by conjugating Boc-SPD to PLGA-PEG-COOH. Briefly, PLGA-PEG-COOH ( $2 \mathrm{~g}, 0.1 \mathrm{mmoL})$ was activated by above EDC/ NHS system for $12 \mathrm{~h}$. After purified and dried, the PLGA-PEGNHS ( $1 \mathrm{~g}, 0.05 \mathrm{mmoL}$ ) dissolved in DCM (4 mL) was followed by the addition of DIEA ( $26 \mathrm{mg}, 0.2 \mathrm{mmoL}$ ) and Boc-SPD (26 mg, $0.075 \mathrm{mmoL})$ dissolved in DCM $(2 \mathrm{~mL})$ in a drop-wise manner with gentle stirring. The reaction was carried out for $24 \mathrm{~h}$ under nitrogen atmosphere and the resultant solution was precipitated and repeatedly washed in ice-cold diethyl and diethyl ether-methanol $(7 / 3, v / v)$. After dried, the block copolymer dissolved in DCM (4 mL) was mixed with trifluoroacetic acid (TFA, $1 \mathrm{mM}$ ) and adjusted the $\mathrm{pH}$ to 8 with a saturated sodium bicarbonate solution to remove of BOC protection. The final SPD-PEG-PLGA block copolymer was dried and stored at $-20{ }^{\circ} \mathrm{C}$ until use.

The ${ }^{1} \mathrm{H}$ NMR spectra of the PEG-PLGA and SPD-PEG-PLGA were recorded at RT using a Bruker Avance $400 \mathrm{MHz}$ NMR spectrometer (Varian, USA) to verify PEG and SPD coupling occurred.

\subsection{Preparation of nanoparticles}

SPD-PEG-PLGA nanoparticles loaded with DOX (SPD-DOXNPs) were prepared by the emulsification-solvent evaporation method. ${ }^{25,26}$ Briefly, SPD-PEG-PLGA (35 mg) and DOX (1.75 mg) were dissolved in $1 \mathrm{~mL}$ DCM. The organic phase was mixed with $2 \%$ PVA aqueous solution $(4 \mathrm{~mL})$ and emulsified by sonication $(120 \mathrm{~W}, 3 \mathrm{~min})$ on ice bath using probe sonication (Emerson Electric Holding, USA). The resulted $\mathrm{O} / \mathrm{W}$ emulsion was further stirred at $30{ }^{\circ} \mathrm{C}$ for $4 \mathrm{~h}$ to evaporated the organic solvent (Scheme 1). The redundant materials and free molecules were removed by centrifugation $\left(20000 \mathrm{~g}, 4{ }^{\circ} \mathrm{C}\right)$ for $30 \mathrm{~min}$ and then resuspended in PBS buffer $(\mathrm{pH} 7.4)$ to obtain a final desired 


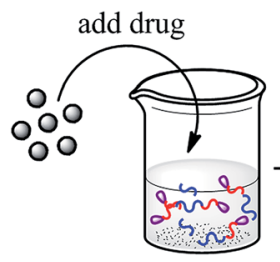

Oil phase

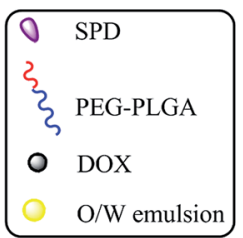

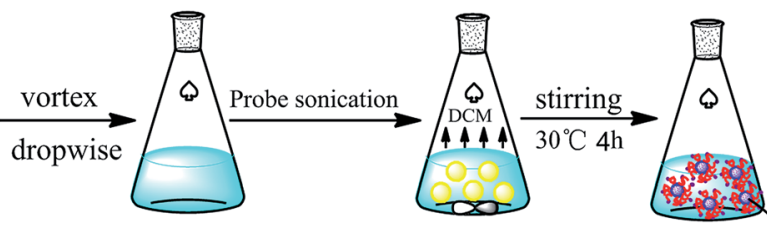

$\mathrm{O} / \mathrm{W}$ emulsion

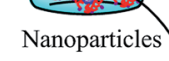

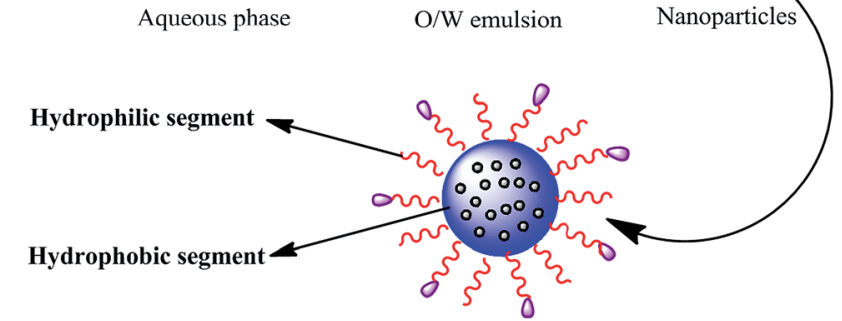

Scheme 1 Construction schematic of SPD-DOX-NPs. DOX and SPD-PEG-PLGA were dissolved in DCM as the organic phase and added into the aqueous phase. Subsequently, the SPD-DOX-NPs were prepared by the self-assembly under probe sonication and evaporated the solvent to get stable nanoparticles.

concentration for further use. The DOX-loaded PEG-PLGA nanoparticles (DOX-NPs) were prepared with the same procedure except PEG-PLGA for carrier material.

\subsection{Characterization of nanoparticles}

2.4.1 Particle size, zeta potential and morphology. Particle size and zeta potential of DOX-NPs and SPD-DOX-NPs were determined by dynamic light scattering (DLS) analysis using Malvern Zeta Sizer Nano series (Nano ZS, Malvern Instruments, UK). The morphology of nanoparticles were investigated by transmission electron microscopy (TEM, Titan G2 60-300, FEI, USA) after sample suspended in water and then dropped into Formvar-coated copper grids.

2.4.2 Drug entrapment efficiency (EE) and loading capacity (LC). For determining the drug entrapment efficiency (EE) and loading capacity (LC) of NPs, the nanoparticles were resuspended and ultrasonicated in methanol for $20 \mathrm{~min}$ after separated by centrifugation $(20000 \mathrm{~g})$ for $30 \mathrm{~min}$. Another aliquot of the nanoparticles suspension was quantitated to determine the total DOX concentration. The DOX was determined by HPLC (LC 2010A, Shimazu, Japan) using an ODS $\mathrm{C}_{18}(200 \times 4.6 \mathrm{~mm}, 5$ $\mu \mathrm{m}$, Diamonsil, Beijing, China) column with sodium lauryl sulfate (SDS) solution (contain $1.36 \mathrm{~mL} \mathrm{~L}^{-1}$ phosphoric acid): acetonitrile : methanol $(50 / 50 / 6, \mathrm{v} / \mathrm{v})$ as the mobile phase at the flow rate of $1.0 \mathrm{~mL} \mathrm{~min}^{-1}$. The column temperature was maintained at $40{ }^{\circ} \mathrm{C}$, sample injection volume was $20 \mu \mathrm{L}$ and the detection wavelength was $223 \mathrm{~nm}$. The EE and LC were calculated using the following formulas:

$$
\begin{aligned}
& \text { EE }(\%)=\frac{\text { amount of loaded DOX in the NPs }}{\text { total amount of DOX added }} \times 100 \% \\
& \text { LC }(\%)=\frac{\text { amount of loaded DOX in the NPs }}{\text { weight of NPs }} \times 100 \%
\end{aligned}
$$

2.4.3 Quantitative detection of SPD on nanoparticles surface. The amount of SPD on nanoparticles surface was determined by fluorescamine method..$^{27,28}$ Briefly, blank SPD-NPs $(0.5$ $\mathrm{mL})$ mixed with PBS buffer $(0.2 \mathrm{M}, \mathrm{pH} 8.0,350 \mu \mathrm{L})$ was reacted with fluorescamine acetone solution $\left(2.89 \mu \mathrm{moL} \mathrm{mL}^{-1}, 350 \mu \mathrm{L}\right)$ for $20 \mathrm{~min}$. And then the samples were analyzed by fluorescence spectrophotometer (RF5301, Shimadzu, Japan) $\left(\lambda_{\mathrm{ex}}: 390 \mathrm{~nm}, \lambda_{\mathrm{em}}\right.$ : $480 \mathrm{~nm})$. The control was used the same approach as described above without NPs. The SPD contents on NPs surface were plotted as a function of the molar quantities of SPD/weight of NPs.

2.4.4 In vitro drug release. The in vitro release behavior of DOX from SPD modified nanoparticles was studied by the membrane diffusion method. Briefly, $1.0 \mathrm{~mL}$ of free DOX solution, DOX-NPs or SPD-DOX-NPs was put into a dialysis bag (MWCO: 3400 ) and then immersed into $20 \mathrm{~mL}$ dissolution medium (PBS 7.4) in a thermostatic shaker vibrated horizontally at $100 \mathrm{rpm}$ and $37^{\circ} \mathrm{C} .1 \mathrm{~mL}$ dissolution medium was taken out for HPLC analysis after dialysis 1, 2, 3, 4, 6, 12, 24, 48, 72, 96, 120, 144, 168, $192 \mathrm{~h}$ and an equal volume of fresh PBS was added immediately.

\subsection{Cell culture}

A549 cells were cultured in DMEM complete medium (containing $10 \%(\mathrm{v} / \mathrm{v})$ FBS and $1 \%$ penicillin/streptomycin (100 U $\left.\mathrm{mL}^{-1}\right)$ ) at $37{ }^{\circ} \mathrm{C}$ in a humidified atmosphere with $5 \% \mathrm{CO}_{2}$, and subcultivated every $2-3$ days at $80-90 \%$ confluence and digested with $0.25 \%$ trypsin-EDTA at a split ratio of $1: 2$.

\subsection{In vitro cellular uptake study}

2.6.1 Qualitative and quantitative analysis on cellular uptake. Fluorescence microscope was utilized to observe the uptake of DOX-loaded nanoparticles by A549 cells. Briefly, A549 cells were seeded and cultured in 24-well culture plate at the density of $4 \times 10^{4}$ cells per well for $24 \mathrm{~h}$. After removed the medium and washed thrice with PBS, $1 \mathrm{~mL}$ of free DOX, DOXNPs, or SPD-DOX-NPs in FBS-free DMEM with a final concentration of $5 \mu \mathrm{g} \mathrm{mL} \mathrm{m}^{-1}$ of DOX was added and incubated for $1 \mathrm{~h}$, 
respectively. Then A549 cells were washed thrice with cold PBS and treated with $4 \%$ paraformaldehyde at room temperature for $20 \mathrm{~min}$ and subsequently stained with $500 \mu \mathrm{L}$ of DAPI $(1 \mu \mathrm{g}$ $\mathrm{mL}^{-1}$ ) for $10 \mathrm{~min}$. After rinsed thrice with PBS, the uptake of various DOX formulations were finally visualized by fluorescence microscope (Ti-S, Nikon, Japan).

For quantitative cellular uptake experiment, A549 cells were seeded in six-well culture plate at the density of $2 \times 10^{5}$ cells per well for $24 \mathrm{~h}$. After washed and incubated by $2 \mathrm{~mL}$ of free DOX, DOX-NPs, or SPD-DOX-NPs solution (with $5 \mu \mathrm{g} \mathrm{mL}{ }^{-1}$ of DOX) for $1 \mathrm{~h}$, respectively. The cells were washed thrice with cold PBS, trypsinized with $0.5 \mathrm{~mL}$ of trypsin-EDTA solution and harvested by centrifuged at $800 \mathrm{rpm}$ for $5 \mathrm{~min}$. After resuspended in 0.5 $\mathrm{mL}$ of PBS, the cellular uptake of various DOX formulations by A549 cells were measured by flow cytometry (FACSVerse, BD, USA), with 10000 events collected in each sample.

2.6.2 Pretreatment with SPD or DFMO. The A549 cells were seeded and cultured in six-well plate at the density of $2 \times 10^{5}$ cells per well for $24 \mathrm{~h}$. After washed thrice with PBS, $2 \mathrm{~mL}$ of free $\operatorname{SPD}\left(0.5,5,50 \mu \mathrm{g} \mathrm{mL}^{-1}\right)$ was added and incubated for $0.5 \mathrm{~h}$. For DFMO pretreatment, the A549 cells were incubated by DFMO $(50,500,5000 \mu \mathrm{M})$ for $24 \mathrm{~h}$. After rinsing thrice with PBS, $2 \mathrm{~mL}$ of DOX-NPs, or SPD-DOX-NPs in FBS-free DMEM (with $5 \mu \mathrm{g}$ $\mathrm{mL}^{-1}$ of DOX) was added and incubated for $1 \mathrm{~h}$. The cells were washed thrice with cold PBS, trypsinized with $0.5 \mathrm{~mL}$ of trypsinEDTA solution and harvested by centrifuged at $800 \mathrm{rpm}$ for 5 min. After resuspended in $0.5 \mathrm{~mL}$ of PBS, A549 cells were analysed by FACS as described above.

2.6.3 The cellular uptake of NPs with various surface SPD contents. The A549 cells were seeded and cultured in six-well plate at the density of $2 \times 10^{5}$ cells per well for $24 \mathrm{~h}$. After washed thrice with PBS, $2 \mathrm{~mL}$ of SPD-DOX-NPs with various target SPD contents $\left(0,20,40,50,60 \mu \mathrm{moL} \mathrm{g}^{-1}\right)$ was added and incubated for $1 \mathrm{~h}$, respectively. Then the cells was washed and analysed by FACS as described above.

\subsection{In vitro antitumor activity evaluation}

The in vitro antitumor activity of the NPs was evaluated using an MTT assay. A549 cells were seeded and cultured in 96-well plate at the density of $4 \times 10^{3}$ cells per well for $24 \mathrm{~h}$. After washed thrice with PBS, $100 \mu \mathrm{L}$ of DMEM complete medium containing free DOX, DOX-NPs, or SPD-DOX-NPs at different DOX concentrations $\left(0.01,0.05,0.1,0.5,1,5,10 \mu \mathrm{g} \mathrm{mL}{ }^{-1}\right)$ was added and incubated for $48 \mathrm{~h}$ in quadruplicate. Then $20 \mu \mathrm{L}$ MTT $\left(0.5 \mathrm{mg} \mathrm{mL}^{-1}\right)$ solution was added to each well and cells were incubated for another $4 \mathrm{~h}$. The MTT containing media were removed and $100 \mu \mathrm{L}$ DMSO was added to dissolve the formazan crystals. The plate was shaked for $10 \mathrm{~min}$ at room temperature. Absorbance was measured at $490 \mathrm{~nm}$ with the multimode reader (Infinite $\mathrm{M} 200$, Tecan, $\mathrm{CH}$ ) and the cell inhibition ratio was calculated.

\subsection{Statistical analysis}

All the experiments were performed in triplicate and data was indicated as mean $\pm \mathrm{SD}$. One-way analysis of variance was used to determine the statistical significance of differences among multiple groups. And a $P$-values $<0.05$ were considered to be statistically significant.

\section{Results and discussion}

\subsection{Synthesis and characterization of SPD-PEG-PLGA copolymer}

SPD-PEG-PLGA copolymer was synthesized via an EDC/NHS technique. ${ }^{29}$ Briefly, PLGA-COOH was firstly transformed into PLGA-NHS and then reacted with $\mathrm{NH}_{2}-\mathrm{PEG}-\mathrm{COOH}$. The resultant PLGA-PEG-COOH was subsequently activated and conjugated with Boc-SPD, and the $t$-butyloxy carbonyl was removed in the presence of TFA to obtain SPD-PEG-PLGA (Fig. 1A). The successful synthesis of SPD-PEG-PLGA was confirmed by ${ }^{1} \mathrm{H}$ NMR spectrum (Fig. 1B). The sharp peak at $3.6 \mathrm{ppm}$ belonged to the methene $\left(-\mathrm{CH}_{2}\right)$ proton of PEG chain. The characteristic peaks at 1.6, 4.8 and $5.2 \mathrm{ppm}$ were attributed to the methyl $\left(-\mathrm{CH}_{3}\right)$, methine $(-\mathrm{CH})$ and methene $\left(-\mathrm{CH}_{2}\right)$ protons in PLGA segment, respectively. And the peak at 1.4, 1.5, 1.7 and $2.7 \mathrm{ppm}$ were assigned to methene $\left(-\mathrm{CH}_{2}\right)$ protons in SPD chains. The sharp peak of PEG at $3.6 \mathrm{ppm}$ and the characteristic peaks of SPD at 1.4 and $1.5 \mathrm{ppm}$ on product demonstrated the successful synthesis of SPD-PEG-PLGA copolymer.

FTIR spectra confirmed the compositions of synthesized polymer (Fig. 1C). The characteristic bands at 3438 and 2886 $\mathrm{cm}^{-1}$ were attributed to $-\mathrm{OH},-\mathrm{NH}_{2}$ and $\mathrm{C}-\mathrm{H}$ stretching vibration, respectively, and the sharp bands at 1466 and $1113 \mathrm{~cm}^{-1}$ were $\mathrm{C}-\mathrm{H}$ deformation vibration and $\mathrm{C}-\mathrm{O}-\mathrm{C}$ absorption of $\mathrm{NH}_{2}-$ PEG-COOH (Fig. 1C, trace a). The band at $1758 \mathrm{~cm}^{-1}$ belonged to carbonyl group of PLGA-COOH (Fig. 1C, trace b), which was also observed in PEG-PLGA copolymer. The absorption bands at 1635 and $1392 \mathrm{~cm}^{-1}$ of amide unit confirmed the successful conjugation of PEG with PLGA (Fig. 1C, trace c). Moreover, the infrared spectra at $1758-1759 \mathrm{~cm}^{-1}(\mathrm{C}=\mathrm{O}$ stretch), 1177-1133 and $1096-1091 \mathrm{~cm}^{-1}$ (C-O and $\mathrm{C}-\mathrm{C}$ stretch) of PEG-PLGA polymer exhibited shifting and intensity increase upon SPD functionalization (Fig. 1C, trace e). The bands changes were attributed to the hydrophilic interactions of primary amine groups in polyamine with PEG-PLGA polymer skeleton. ${ }^{30,31}$

\subsection{Preparation and characterization of nanoparticles}

DOX-loaded nanoparticles was prepared by a typical emulsification-solvent evaporation method (Scheme 1). Briefly, the drug and synthesized polymers were first dissolved in DCM and quickly added into the aqueous phase. The $\mathrm{O} / \mathrm{W}$ emulsions were transformed into uniform nano-droplets under probe sonication and evaporated the solvent to get stable nanoparticles. The as-prepared nanoparticles were characterized for the hydrodynamic size, polydispersity and zeta potential. Both the average diameter of DOX-NPs and SPD-DOX-NPs were about $151 \mathrm{~nm}$ with homogenous size distribution observed by DLS (PDI $<0.1$, Fig. 2A and B). The morphology of the NPs was then characterized by transmission electron microscope (TEM). As showed in Fig. 2A and B, DOX-NPs or SPD-DOX-NPs exhibited relatively monodispersed spherical shapes with a uniform size and a smooth surface. The intact morphologies of nanoparticles 


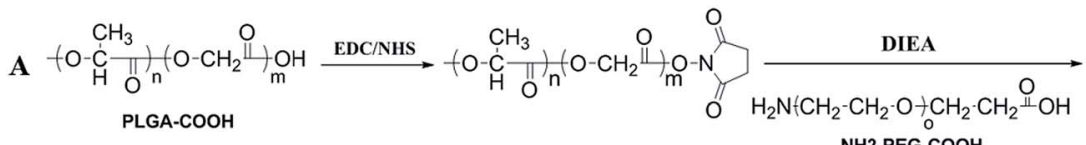

$$
\begin{aligned}
& \text { NH2-PEG-COOH }
\end{aligned}
$$

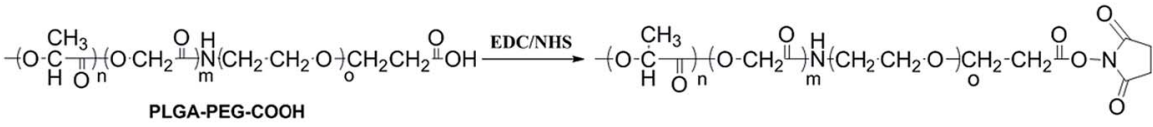

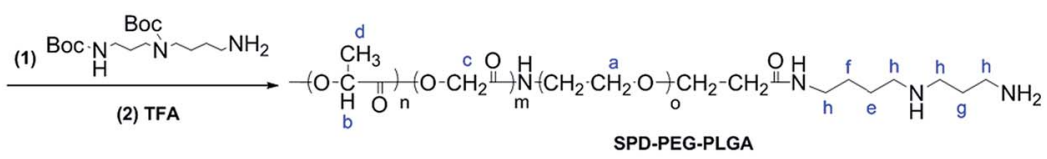

B

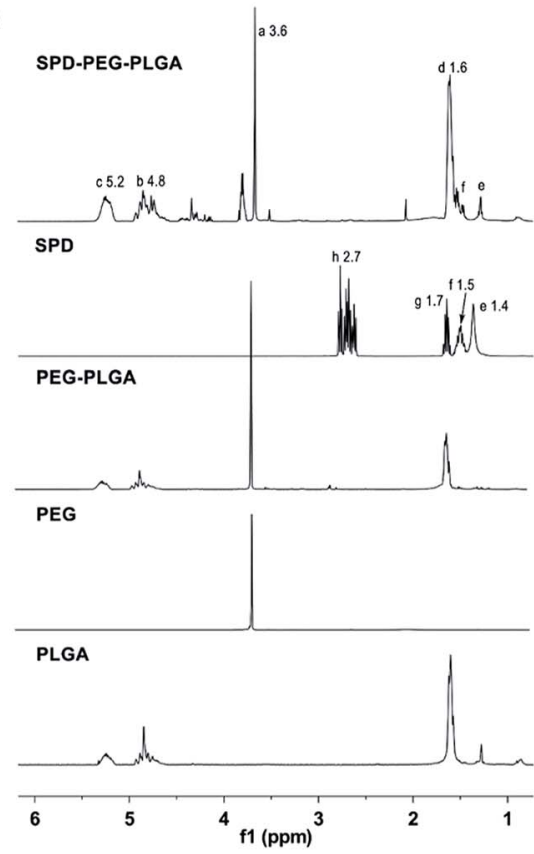

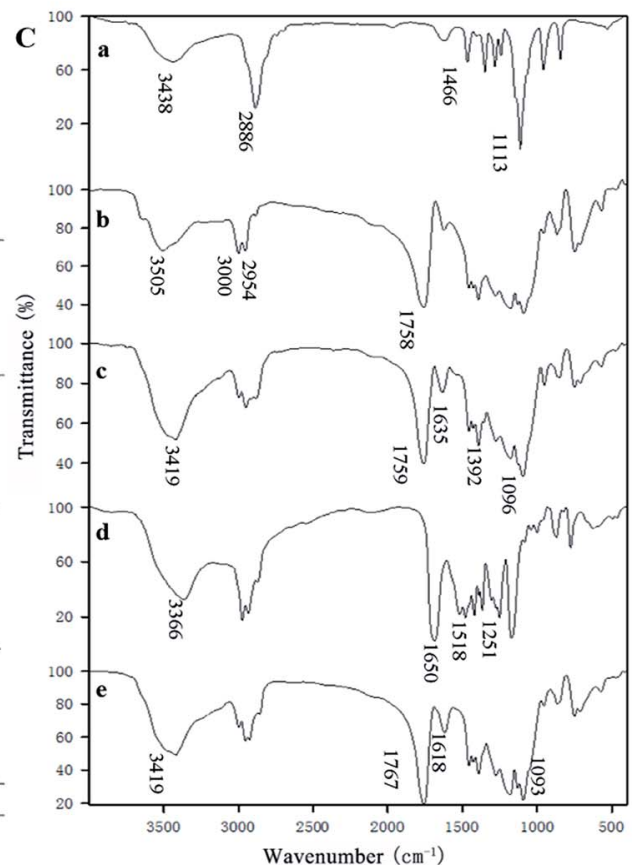

Fig. 1 Synthesis and characterization of SPD-PEG-PLGA copolymer. (A) Synthetic schemes of PEG-PLGA and SPD-PEG-PLGA. (B) ${ }^{1} \mathrm{H}$ NMR spectra of PLGA-COOH, $\mathrm{H}_{2} \mathrm{~N}-\mathrm{PEG}-\mathrm{COOH}, \mathrm{SPD}$ and the synthesized PLGA-PEG-COOH and SPD-PEG-PLGA copolymer in CDCl 3 . (C) FTIR spectra of $\mathrm{H}_{2} \mathrm{~N}-\mathrm{PEG}-\mathrm{COOH}$ (a), PLGA-COOH (b), PLGA-PEG-COOH (c), SPD (d) and SPD-PEG-PLGA copolymer (e).

provided a stable carrier support for drug encapsulation. Notably, the size of DOX-loaded NPs determined by TEM was slightly smaller than that measured by DLS, which could be ascribed to the swelling or stretching of the SPD-PEG chain in solution for DLS measurement, while shrinking and crimping on the copper grid surface during drying and vacuumizing for TEM characterization. ${ }^{32,33}$

The zeta potential of DOX-NPs was observed to be about $-5.2 \mathrm{mV}$. After SPD modification, the zeta potential of SPDDOX-NPs was characterized to be nearly $-2.4 \mathrm{mV}$. The decreased negative charge can be attributed to the fact that the electropositive SPD partly screened the negatively charged carboxyl groups tails on PEG-PLGA polymer. The EE and of LC of optimized DOX-NPs were calculated to be $56.4 \%$ and $0.83 \%$, respectively. For SPD-DOX-NPs, a slightly decreased EE and LC were observed to be $50.1 \%$ and $0.78 \%$, respectively. This may be due to the increased hydrophilicity of PLGA-PEG block copolymer after terminal conjugated with spermidine (SPD) hydrophilic chain, and the hydrophobic DOX tended to diffuse from nanoparticles to aqueous solvent due to the reduced binding affinity of DOX with SPD-PEG-PLGA during SPD-DOX-NPs preparation.

The amount of SPD on nanoparticles surface was determined by a fluorescamine-based assay (Fig. S1A $\dagger$ ). In this assay, the fluorescamine agent could generate a fluorescent compound ( $\lambda_{\text {ex }}: 390 \mathrm{~nm}, \lambda_{\text {em }}: 480 \mathrm{~nm}$ ) in the presence of primary amine at pH 9.0 with extremely high sensitivity on the pM scale, while the excess reagents generate hydrolysis products with nonfluorescent. ${ }^{34}$ As showed in Fig. S1B and $\mathrm{C}, \dagger$ the fluorescence intensity of fluorescamine at $480 \mathrm{~nm}$ was linear correlation with the concentration of Boc-SPD. Based on the calibration curve, the surface SPD content was measured to be $46.4 \mu \mathrm{moL} \mathrm{g}^{-1}$.

\subsection{In vitro drug release}

In vitro drug release of DOX from DOX-loaded NPs at $37^{\circ} \mathrm{C}$ was evaluated by a dialysis method at $\mathrm{pH} 7.4$ for up to $192 \mathrm{~h}$ 

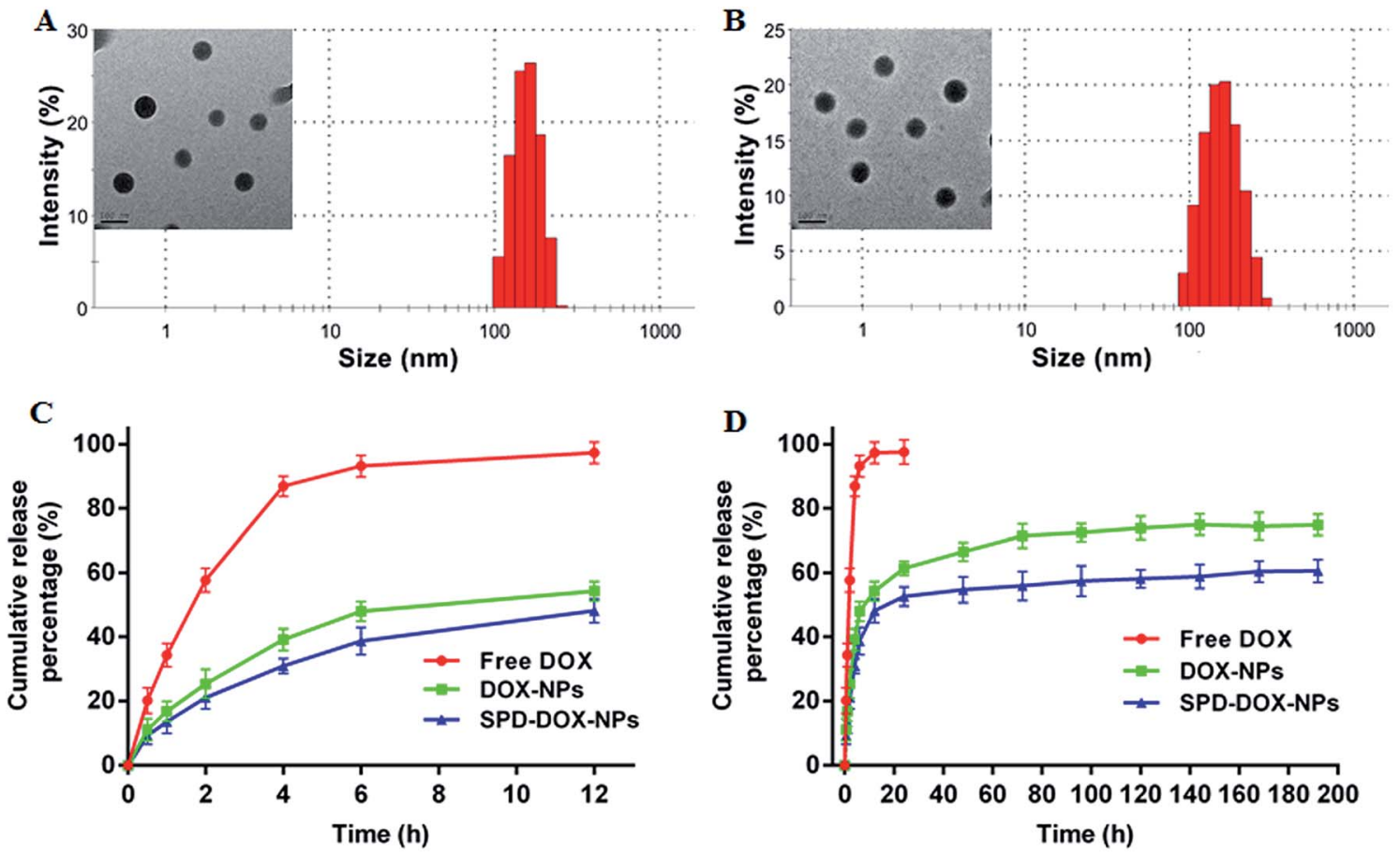

Fig. 2 Particle size distribution and TEM images of DOX-NPs (A) and SPD-DOX-NPs (B), respectively. In vitro release profiles of DOX from DOXNPs and SPD-DOX-NPs in PBS (pH 7.4) with $0.05 \%$ SDS at $37^{\circ} \mathrm{C}$ for $12 \mathrm{~h}(\mathrm{C})$ and $192 \mathrm{~h}(\mathrm{D})$. Data were shown as mean \pm SD $(n=3)$.

incubation. As showed in Fig. 2C, about $90 \%$ free DOX diffused to release medium within $4 \mathrm{~h}$ and almost released totally after $6 \mathrm{~h}$. While DOX released from DOX-NPs or SPD-DOX-NPs revealed a remarkably slower release, and the two nanoparticles exhibited a similar release behaviors during the whole experiment period. An initial burst of DOX release from DOXNPs or SPD-DOX-NPs was observed in the first $12 \mathrm{~h}(\sim 50 \%)$ due to a large amount of drug adsorbed on the surface of NPs. After the sudden release period, the release of DOX from NPs was sustained over following $192 \mathrm{~h}$ (Fig. 2D). In addition, a series of SPD-PEG-PLGA polymers with different SPD contents from 20 to $60 \mu \mathrm{moL} \mathrm{g}{ }^{-1}$ have also been synthesized to prepare SPD-DOX-NPs (Table 1). The cumulative release of DOX from SPD-DOX-NPs was gradually decreased with increased SPD contents (Fig. S2 $\dagger$ ). This can be explained by that terminal carboxyl groups of PEG chains are more labile to hydrolysis than that of amide bonded SPD chains, leading to faster erosioncontrolled drug release from NPs. The phenomenon was also observed in Fig. 2C and D, as the cumulative release of DOX from DOX-NPs (nearly 75\%) was significantly increased compared with that of SPD-DOX-NPs (nearly 61\%) in $192 \mathrm{~h}$.

\subsection{Cellular uptake of DOX loaded nanoparticles}

The fluorescence microscope was used to visualize the cellular uptake of free DOX and DOX loaded nanoparticles by A549 cells at an equivalent $5 \mu \mathrm{g} \mathrm{mL} \mathrm{m}^{-1}$ of DOX. The cells were imaged in the red channel for DOX and the blue channel for the nuclear stain. Among various groups, the brightest fluorescence was observed when A549 cells were incubated with SPD-DOX-NPs (Fig. 3A-C), indicating that the modification of SPD on the nanoparticles surface facilitates the cellular uptake of NPs. By contrast, free DOX solution exhibited weak fluorescence and DOX-NPs with only moderate fluorescence.

Quantitative evaluation of cellular uptake of various groups was performed by flow cytometry after $1 \mathrm{~h}$ incubation. As showed in Fig. 3D and E, the fluorescence intensity of SPDDOX-NPs was significantly enhanced compared to free DOX $(P<$ $0.01)$ or DOX-NPs $(P<0.05)$, indicating the active uptake of SPDmodified NPs via affinity binding with PTS overexpressed on A549 cells surface. Meanwhile, though the cellular uptake of DOX-NPs was attributed to the endocytosis of NPs and free diffusion of DOX adsorbed on NPs surface, leading the mean fluorescence intensity of DOX-NPs enhanced $\sim 1.1$ fold

Table 1 Characterization of nanoparticles with various surface SPD contents (mean $\pm S D, n=3$ )

\begin{tabular}{|c|c|c|c|c|}
\hline SPD contents $\left(\mu \mathrm{mol} \mathrm{g}{ }^{-1}\right)$ & Size (nm) & Zeta potential (mV) & $\mathrm{EE}(\%)$ & LC $(\%)$ \\
\hline 0 & $151.9 \pm 3.6$ & $5.2 \pm 1.4$ & $56.43 \pm 1.97$ & $0.83 \pm 0.08$ \\
\hline 20 & $152.2 \pm 2.0$ & $4.0 \pm 0.7$ & $55.15 \pm 1.50$ & $0.78 \pm 0.02$ \\
\hline 50 & $150.5 \pm 1.0$ & $2.3 \pm 1.2$ & $50.80 \pm 1.13$ & $0.82 \pm 0.06$ \\
\hline 60 & $151.7 \pm 1.9$ & $1.4 \pm 0.5$ & $50.16 \pm 1.11$ & $0.78 \pm 0.05$ \\
\hline
\end{tabular}



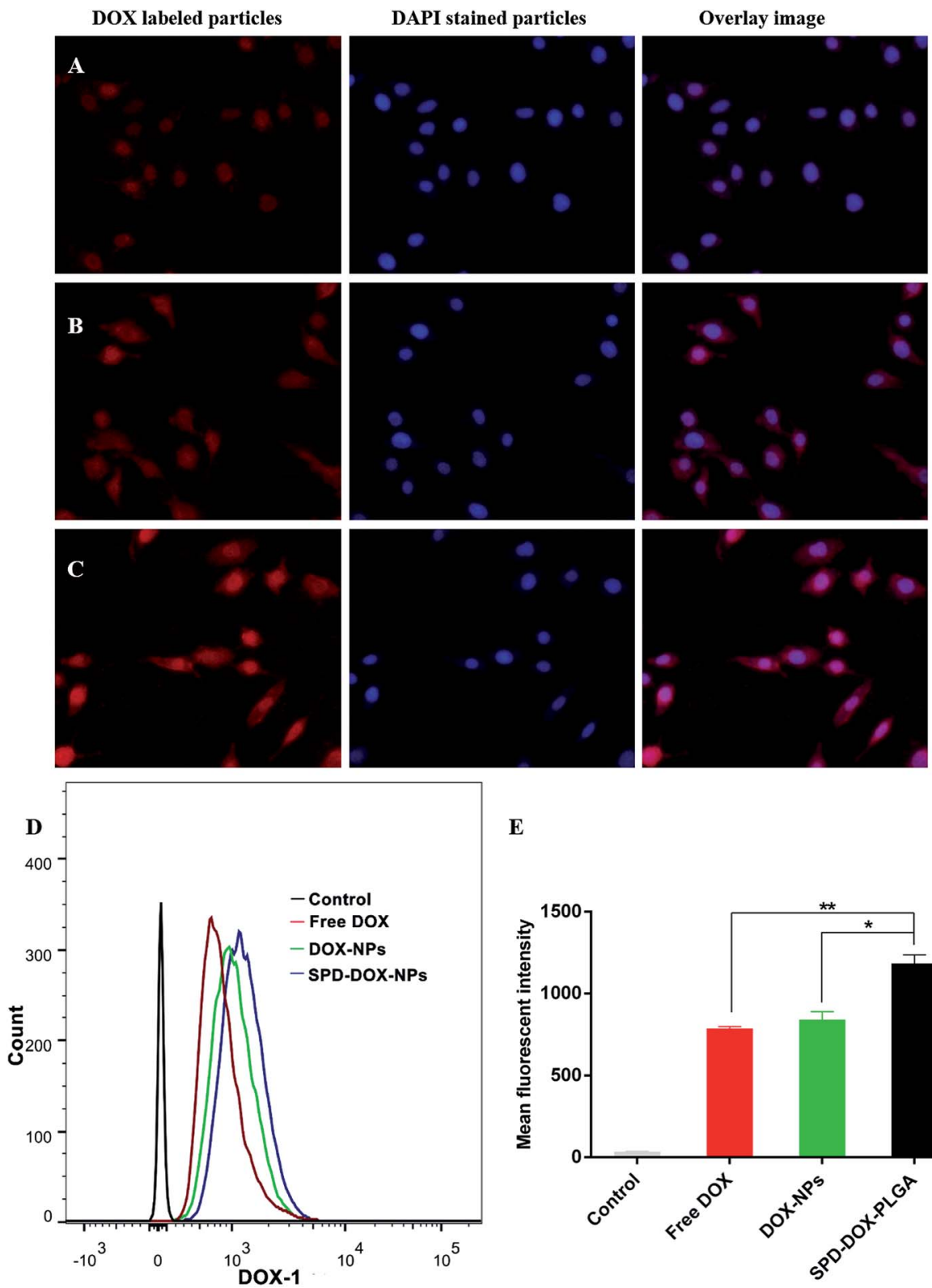

$\mathbf{E}$

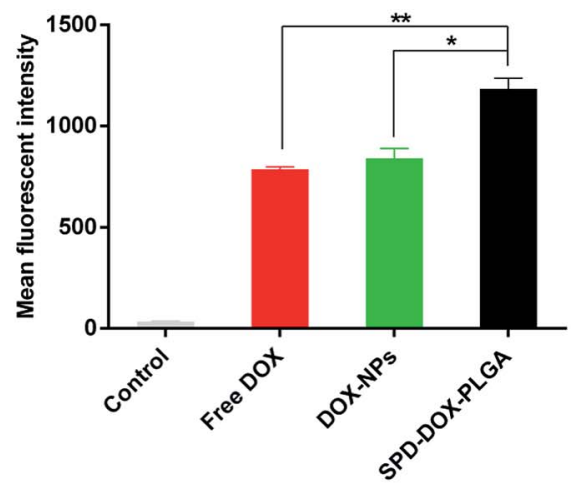

Fig. 3 Fluorescence microscope images of $A 549$ cells incubated with free DOX (A), DOX-NPs (B), and SPD-DOX-NPs (C) at $37^{\circ} \mathrm{C}$ for $1 \mathrm{~h}$. The final DOX concentration was $5 \mu \mathrm{g} \mathrm{mL}^{-1}$. Cell nuclei were stained blue with DAPI and overlaid with red fluorescence images of DOX. Quantitative analysis results of cellular uptake of various DOX-loaded NPs by flow cytometry ( $D$ and E). Data were presented as mean \pm SD $(n=3), * P<0.01$, SPD-DOX-NPs vs. free DOX; *P<0.05, SPD-DOX-NPs vs. DOX-NPs.

compared with that of free DOX, the cellular uptake of DOX-NPs by passive targeting was not significantly stronger than free DOX $(P>0.05)$.

\subsection{Pretreatment with SPD or DFMO}

To confirm that the enhanced cellular uptake of SPD-DOX-NPs was due to its surface SPD modification, two parallel experiments were design. In the first experiment, the A549 cells were pre-treated with free SPD for $30 \mathrm{~min}$ so that the PTS on tumor cells surface was blocked. In this case, the specific enhancement effect of SPD-DOX-NPs can be abolished if PTS was important for particle uptake. Otherwise, the free SPD pre- treatment would not have much effect on particle internalization. As showed in Fig. 4A and B, the mean fluorescent intensity of A549 cells incubated with DOX-NPs was similar to treated cells with free SPD prior to the addition of NPs. While the internalization efficiency of SPD-DOX-NPs was reduced since the PTS binding sites was gradually occupied by free SPD. And the competitive effect was almost saturated when the amount of free SPD up to be $0.5 \mu \mathrm{g} \mathrm{mL} \mathrm{m}^{-1}$.

In the other experiment, the cells were pre-treated with DFMO, a commonly used reagent to up-regulate the cell uptake of exogenous polyamines. As showed in Fig. 4C and D, the cell uptake of DOX-NPs changed lowness after pretreatment of 

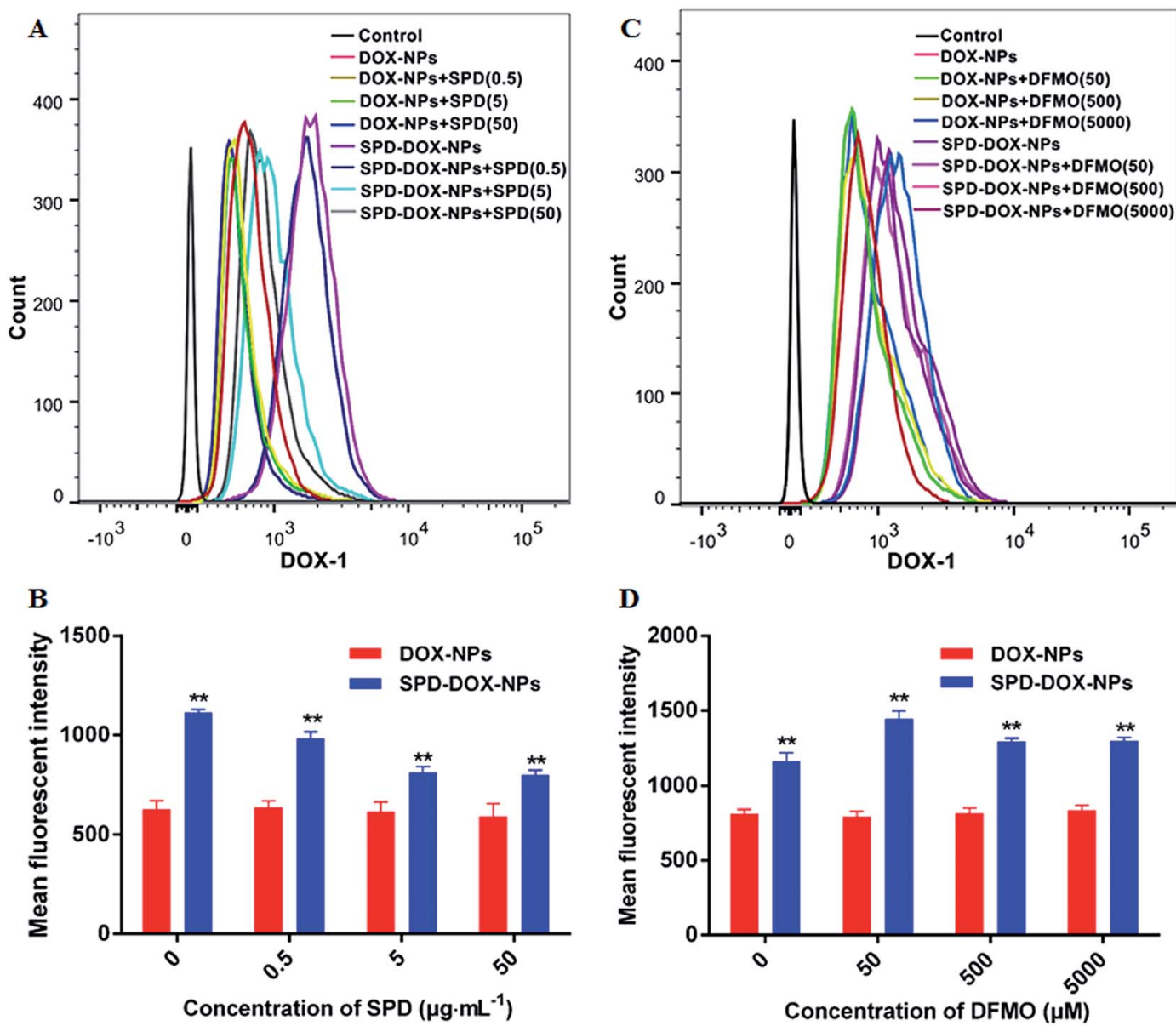

Fig. 4 Effect of SPD or DFMO pre-incubations on A549 cells uptake of DOX-NPs and SPD-DOX-NPs. (A and B) A549 cells was incubated by various concentration of SPD $\left(0-50 \mu \mathrm{g} \mathrm{mL}^{-1}\right)$ for $0.5 \mathrm{~h}$, following cultured by the same concentration of SPD with DOX-NPs or SPD-DOX-NPs for $1 \mathrm{~h}$. (C and D) A549 cells was incubated by various concentration of DFMO $(0-5000 \mu \mathrm{M})$ for $24 \mathrm{~h}$, following cultured by DOX-NPs and SPDDOX-NPs for $1 \mathrm{~h}$. Data were presented as mean \pm SD $(n=3), * * P<0.01$, SPD-DOX-NPs vs. DOX-NPs.

DFMO due to its passive transport. By contrary, the mean fluorescent intensity of A549 cells incubated with SPD-DOXNPs was increased significantly $(P<0.05)$ after DFMO preconditioning for $24 \mathrm{~h}$. In fact, DFMO was as a suicide inhibitor of ornithine decarboxylase (ODC), one of the key enzymes involved in polyamine biosynthesis. ${ }^{35,36}$ The PTS activity of cancer cells was usually upregulated to replenish depleted intracellular polyamine pools, ${ }^{37,38}$ leading more SPD-DOX-NPs transported into cells. However, the internalization efficiency reduced slightly due to the affection of cell viability when the amount of DFMO over $50 \mu \mathrm{M}$ (Fig. S3 $\dagger$ ). Taken together, the parallel experiments of SPD and DFMO pretreatment suggested that SPD-DOX-NPs was transported into cancer cells by a PTSmediated endocytosis process.

\subsection{The cellular uptake of NPs with various surface SPD contents}

In order to investigate the effect of SPD density on cellular uptake efficiency, we synthesized SPD-PEG-PLGA polymers with different SPD contents from 20 to $60 \mu \mathrm{moL} \mathrm{g}^{-1}$. As shown in Table 1 , the variation of SPD contents had little impact on the particle size, drug-encapsulation ration and carried amount. Since the terminal carboxyl was reacted with the positive charge of polyamine skeleton, the zeta potential of SPD-NPs was near to neutrality with the improvement of SPD contents. After co-cultured with A549 cells for $1 \mathrm{~h}$, the fluorescence intensity of the cells increased with the raise of SPD contents (Fig. 5A). Meanwhile, the relative fluorescent intensities of SPD-DOX-NPs with different SPD contents were normalized by that of non-SPD DOX-NPs. As shown in Fig. 5B, higher SPD content resulted in relatively stronger fluorescence intensity, indicating that SPD modification facilitates the internalization of NPs into A549 cells via SPD bound to the PTS overexpressed on the surface of cancer cells. However, the targeting ability of SPD was weakened with the SPD contents over $50 \mu \mathrm{moL} \mathrm{g}^{-1}$. It was presumably caused by the affinity between conjugated SPD and PTS was reduced by the steric hindrance of nanoparticles, or the active sites of SPD were saturated with targeted NPs. These results indicated that the cellular internalization of SPDmodified NPs was receptor-dependent for anti-cancer therapy. 

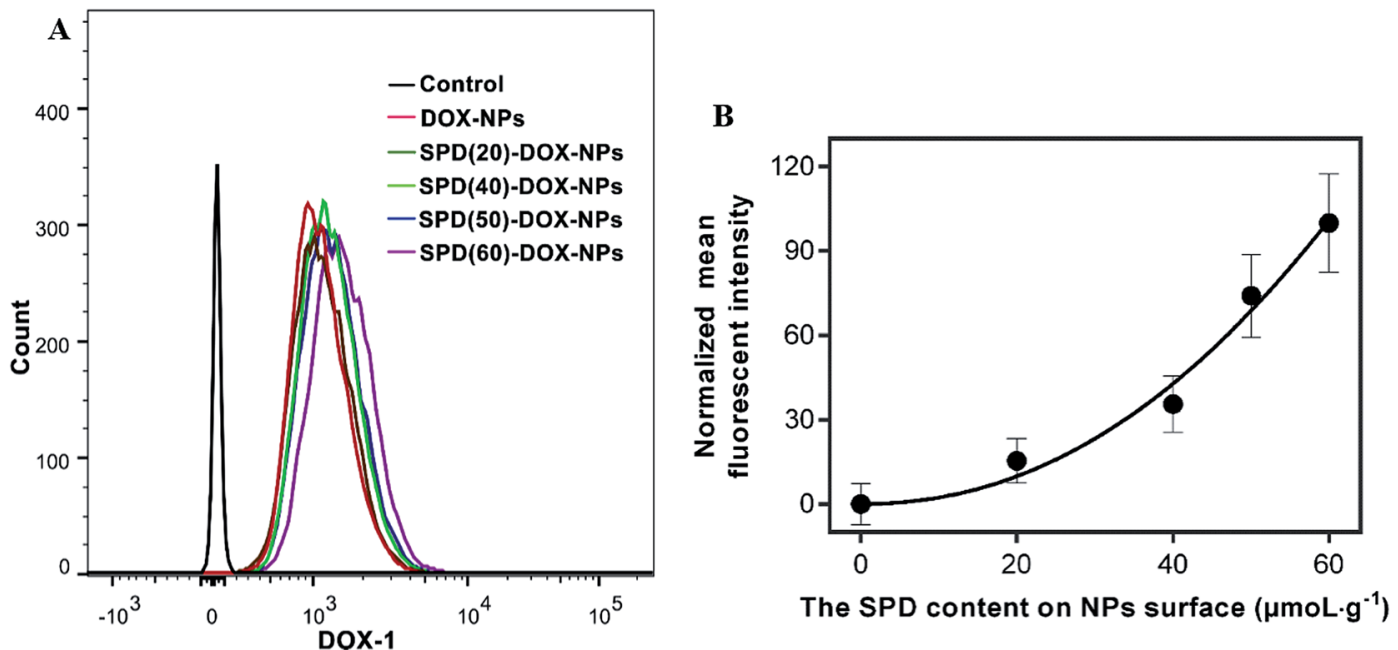

Fig. 5 (A) Cellular uptake of DOX-loaded NPs with different SPD contents on NPs surface in A549 cells for $1 \mathrm{~h}$. (B) The mean fluorescence intensity of SPD-DOX-NPs analyzed by flow cytometry was normalized by that of non-SPD DOX-NPs. Data were shown as mean \pm SD $(n=3)$.

\subsection{In vitro cytotoxicity}

The flow cytometry analysis showed that the uptake of nanoparticles increased by PTS-overexpressing tumor cells after SPD modified on NPs surface. However, the antitumor activity is undefined. To understand the effects of this targeted drug delivery system on cancer cell proliferation, the in vitro antitumor efficacy of various DOX formulations was evaluated in A549 cells by MTT assay. Blank NPs or SPD-NPs did not exhibit obvious cytotoxicity at high concentration of $4 \mathrm{mg} \mathrm{mL}$ (Fig. S4 $\dagger$ ), suggesting that the polymer delivery carriers possess satisfactory biocompatibility, and that cytotoxicity of DOXloaded NPs was mostly caused by the encapsulated drug. As shown in Fig. 6A, the growth inhibition of A549 cells was increased apparently in a concentration- and formulationdependent pattern. As a control, the $\mathrm{IC}_{50}$ value of free DOX was $1.13 \pm 0.11 \mu \mathrm{g} \mathrm{mL} \mathrm{m}^{-1}$. DOX-loaded SPD-free NPs revealed similar cytotoxicity with $\mathrm{IC}_{50} 0.89 \pm 0.16 \mu \mathrm{g} \mathrm{mL} \mathrm{m}^{-1}$ compared to free DOX for A549 cells $(P>0.05)$. Meanwhile, the toxicity of
SPD-DOX-NPs increased gradually with rising contents of surface SPD. At the highest SPD contents, the cytotoxicity reached 1.6-fold and 2.0-fold compared the SPD-free and free DOX formulation, respectively (Fig. 6B). Overall, PTS-mediated binding and internalization of SPD-NPs have been proposed to enhance the cancer cells proliferation inhibition of anticancer therapeutic agents.

In fact, PTS was highly overexpressed in multiple tumor cells, implementation of PTS as a targeting site was a potential strategy for targeted delivery of chemotherapeutic agents into tumor cells. In this study, SPD, with moderate positive charge and chain length, was first exploited as a targeting ligand in a PEG-PLGA nanoparticle-based DOX delivery system. Since SPD exhibited high binding affinity to PTS, such a SPD-PEGPLGA delivery system may have the potential to improve the delivery of DOX to cancer cells. Our results suggested that SPDDOX-NPs could actively targeting to cancer cells and offer an effective antitumor efficiency compared to free DOX and DOX-NPs.
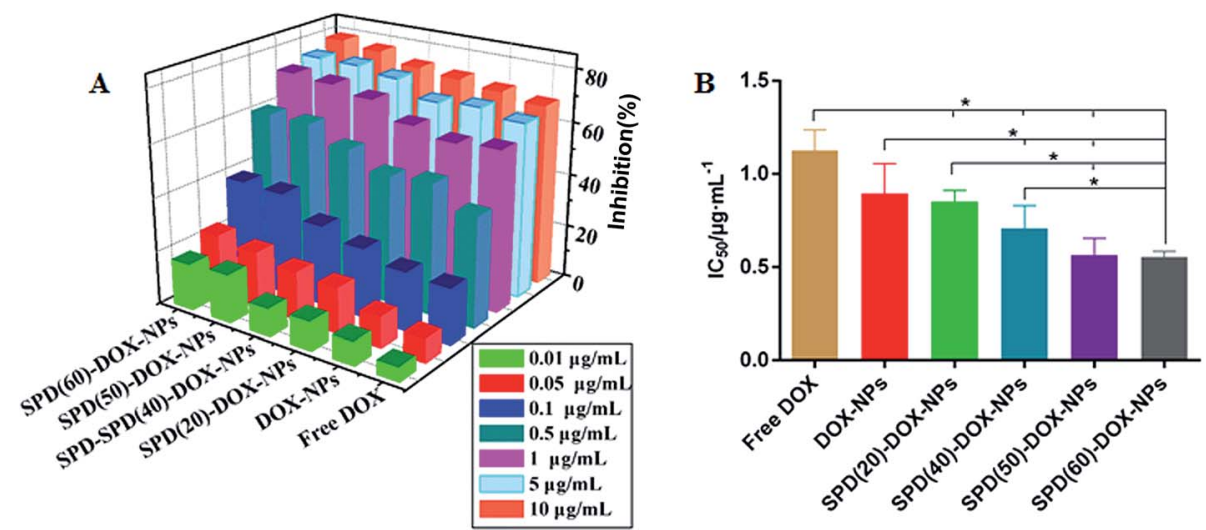

Fig. 6 (A) In vitro cytotoxicity of various DOX formulations on A549 cells treated for $48 \mathrm{~h}$ with free DOX solution, DOX-NPs and SPD-DOX-NPs. (B) IC 50 values of DOX, DOX-NPs and SPD-DOX-NPs on A549 cells. Data were shown as mean \pm SD $(n=4)$. 


\section{Conclusion}

In this work, DOX-loaded SPD-PEG-PLGA nanoparticles were constructed and characterized. The NPs formulation was uniform spherical with sustained drug release profile, enhanced cellular uptake and thereby cytotoxicity in a concentration dependent manner with SPD contents on NPs surface. All of these results suggested that these SPD modified PEG-PLGA nanoparticle-based drug delivery system might be a promising drug carrier for targeting treatment strategy of cancers.

\section{Conflict of interest}

The authors declare no conflicts of interest. The authors alone are responsible for the content and writing of this article.

\section{Acknowledgements}

This work was supported by National Natural Science Foundation of China (81603061, 81573374), Graduate student independent exploration and Innovation Foundation of Central South University (2016zzts495). We thank the Analysis and Testing Center of Central South University for assistance on the ${ }^{1} \mathrm{H}$ NMR characterization of SPD-PEG-PLGA copolymer and TEM imaging of nanoparticles. We also thank the Department of Pathology of Hsiang-Ya Medical College for assistance on the flow cytometry services.

\section{References}

1 R. L. Siegel, K. D. Miller and A. Jemal, Ca-Cancer J. Clin., 2016, 66, 7-30.

2 R. Z. C. Academy and C. Acad, Ca-Cancer J. Clin., 2016, 66, 115-132.

3 M. Heron, D. Ph, R. N. Anderson and D. Ph, NCHS Data Brief, 2016, vol. 254, pp. 1-8.

4 B. Colagiuri, H. Dhillon, P. N. Butow, J. Jansen, K. Cox and J. Jacquet, J. Pain Symptom Manage., 2013, 46, 275-281.

5 M. E. Godsey, S. Suryaprakash and K. W. Leong, RSC Adv., 2013, 3, 24794-24811.

6 S. Kumar and P. Maiti, RSC Adv., 2016, 6, 67449-67480.

7 S. Acharya and S. K. Sahoo, Adv. Drug Delivery Rev., 2011, 63, 170-183.

8 I. Bala and S. Haribaran, Crit. Rev. Ther. Drug Carrier Syst., 2004, 21, 387-422.

9 S. M. Ryan, G. Mantovani, X. Wang, D. M. Haddleton and D. J. Brayden, Expert Opin. Drug Delivery, 2008, 5, 371-383.

10 S. Sharma, A. Parmar, S. Kori and R. Sandhir, TrAC, Trends Anal. Chem., 2015, 80, 30-40.

11 M. Das, C. Mohanty and S. K. Sahoo, Expert Opin. Drug Delivery, 2009, 6, 285-304.

12 E. Agostinelli, F. Vianello, G. Magliulo, T. Thomas and T. J. Thomas, Int. J. Oncol., 2015, 46, 5-16.

13 S. D. Steichen, M. Caldorera-moore and N. A. Peppas, Eur. J. Pharm. Sci., 2013, 48, 416-427.
14 C. Moinard, L. Cynober and J. P. de Bandt, Clin. Nutr., 2005, 24, 184-197.

15 S. Tomasi, J. Renault, B. Martin, S. Duhieu, V. Cerec, M. Le Roch, P. Uriac and J. G. Delcros, J. Med. Chem., 2010, 53, 7647-7663.

16 C. Wang, J. Delcros, J. Biggerstaff and O. Phanstiel, J. Med. Chem., 2003, 46, 2663-2671.

17 A. Muth, J. Kamel, N. Kaur, A. C. Shicora, I. S. Ayene, S. K. Gilmour and O. Phanstiel, J. Med. Chem., 2013, 56, 5819-5828.

18 S. L. Nowotarski, P. M. Woster and R. A. Casero, Expert Rev. Mol. Med., 2013, 15, e3.

19 S. Q. Xie, J. H. Wang, H. X. Ma, P. F. Cheng, J. Zhao and C. J. Wang, Toxicology, 2009, 263, 127-133.

20 A. J. Palmer and H. M. Wallace, Amino Acids, 2010, 38, 415422.

21 S. T. Matalon, A. Ornoy and M. Lishner, Reprod. Toxicol., 2004, 18, 219-230.

22 B. Chaveli-López, J. clin. exp. dent., 2014, 6, 3-5.

23 S. Piper and T. McDonagh, Future Cardiol., 2015, 11, 453470.

24 K. Krukiewicz and J. K. Zak, Mater. Sci. Eng., C, 2016, 62, 927942.

25 R. A. Jain, Biomaterials, 2000, 21, 2475-2490.

26 C. Pinto Reis, R. J. Neufeld, A. J. Ribeiro and F. Veiga, Nanomed. Nanotech. Biol. Med., 2006, 2, 8-21.

27 J. M. Chalovich and E. Eisenberg, Nanotechnology, 2013, 24, 355101.

28 F. Badalà, K. Nouri-mahdavi and D. A. Raoof, ACS Nano, 2012, 6, 512-522.

29 M. Alibolandi, M. Ramezani, F. Sadeghi, K. Abnous and F. Hadizadeh, Int. J. Pharm., 2015, 479, 241-251.

30 S. M. Alex, M. R. Rekha and C. P. Sharma, Int. J. Pharm., 2011, 410, 125-137.

31 S. Sanyakamdhorn, P. Chanphai and H. A. Tajmir-Riahi, J. Photochem. Photobiol., B, 2014, 130, 30-39.

32 J. Li, Y. Zhou, C. Li, D. Wang, Y. Gao, C. Zhang, L. Zhao, Y. Li, Y. Liu and X. Li, Bioconjugate Chem., 2015, 26, 110-119.

33 N. Li, X. R. Li, Y. X. Zhou, W. J. Li, Y. Zhao, S. J. Ma, J. W. Li, Y. J. Gao, Y. Liu, X. L. Wang and D. D. Yin, Biomaterials, 2012, 33, 8881-8892.

34 S. Udenfriend, S. Stein, P. Böhlen, W. Dairman, W. Leimgruber and M. Weigele, Science, 1972, 178, 871-872. 35 P. A. Sample, C. Boden, Z. Zhang, J. Pascual, T. Lee, L. M. Zangwill, R. N. Weinreb, J. G. Crowston, E. M. Hoffmann, F. A. Medeiros, T. Sejnowski and M. Goldbaum, Cancer Prev. Res., 2014, 7, 1198-1209.

36 F. L. Meyskens and E. W. Gerner, Clin. Cancer Res., 1999, 5, 945-951.

37 A. Muth, M. Madan, J. J. Archer, N. Ocampo, L. Rodriguez and O. Phanstiel, J. Med. Chem., 2014, 57, 348-363.

38 H. M. Wallace and A. V Fraser, Amino Acids, 2004, 26, 353365. 